\section{Cooperación internacional ¿en qué estamos y hacia dónde vamos?}

\author{
EDUARDO KATTAN ${ }^{1,2,3, a}$, ARON KUROIWA ${ }^{4, b}$, RODRIGO LÓPEZ $^{2,5, \mathrm{c}}$
}

\section{International cooperation. Where are we, and where are we heading to?}

Unmet needs in global health are important issues, not yet solved by the international community. A variety of individuals, non-governmental organizations (NGO) and government institutions have tried to address this situation, developing multiple types of international cooperation (IC), such as humanitarian aid (HA), cooperation for development (CD) and medical missions (MM). In the last decades, we have witnessed an exponential growth in the creation and participation of these projects. Moreover, in the last 20 years, Chile has experimented a real paradigm switch, from a receiver to a provider of IC. Due to the recent surge in interest and relevance of the topic, we performed a narrative revision of the literature related with IC. In the present article, we characterize the different types of IC, with emphasis in MM: we address the risks, controversies and ethical problems associated with these activities. We finally propose some guidelines for the future development and promotion of $M M$.

(Rev Med Chile 2017; 145: 783-789)

Key words: Developing Countries; International Cooperation; Medical Missions, Official; Volunteers.
'Departamento de Medicina Intensiva. Facultad de Medicina, Pontificia Universidad Católica de Chile. Santiago, Chile. ${ }^{2}$ División de Anestesiología. Facultad de Medicina, Pontificia Universidad Católica de Chile. Santiago, Chile.

${ }^{3}$ Centro de Educación Médica. Facultad de Medicina, Pontificia Universidad Católica de Chile. Santiago, Chile.

${ }^{4}$ Departamento de Traumatología y Ortopedia. Facultad de Medicina, Pontificia Universidad Católica de Chile. Santiago, Chile.

${ }^{5}$ Centro de Bioética. Facultad de Medicina, Pontificia Universidad Católica de Chile.

Santiago, Chile.

aResidente de Medicina Intensiva. ${ }^{\mathrm{b}}$ Residente de Traumatología y Ortopedia. 'Magíster en Bioética.

No se recibieron fondos para la realización de este trabajo.

Autores no declaran conflicto de intereses.

Recibido el 6 de noviembre de 2016, aceptado el 13 de junio de 2017.

Correspondencia a:

Dr. Rodrigo López

División de Anestesiología, Centro de Bioética. Facultad de Medicina, Pontificia

Universidad Católica de Chile

Marcoleta 367, Santiago, Chile.

Teléfono: 223543000

ralopez@uc.cl

\section{L}

a Constitución de la Organización Mundial de la Salud (OMS) define a los derechos humanos como garantías jurídicas universales, siendo uno de ellos el buscar el goce del grado máximo de salud que se pueda lograr ${ }^{1}$. Sin embargo, la demanda por prestaciones de salud no está adecuadamente satisfecha en todas las sociedades, de manera incidental o crónica, comprometiendo el nivel de salud de la población, medido por diferentes indicadores, lo que constituye un problema de alto impacto. Actualmente, los países de bajos recursos (PBR), definidos como aquellos con un producto interno bruto (PIB) menor a 1.045 USD/año/persona, comprenden a 621 millones de personas y corresponden principalmente a países de África subsahariana. En estos países se concentran los peores indicadores en salud. A modo de ejemplo, en la Tabla 1 se comparan indicadores en salud de PBR con países de altos recursos (PAR) (PIB mayor 12.736 USD/año/persona $)^{2}$.

Diversos individuos, organizaciones no gubernamentales (ONG) e instituciones de gobierno han intentado combatir esta situación, naciendo la cooperación internacional (CI). Esta se entiende como "la relación que se establece entre dos o más países, organismos u organizaciones de la sociedad civil, con el objetivo de alcanzar metas de desarrollo consensuada"3. Se han desarrollado diversas modalidades de CI, como la ayuda humanitaria (AH), la cooperación para el desarrollo (CD), y los operativos médicos (OM). La AH corresponde al apoyo de poblaciones que han sido víctimas de crisis humanitarias, ya sea por guerras o desastres naturales. La CD consiste en realizar proyectos de cooperación desde las necesidades locales e invo- 
Tabla 1 Comparación de indicadores en salud entre PBR y PAR [2]

\begin{tabular}{|lcc|}
\hline Indicador & PBR & PAR \\
PIB (USD/año/persona) & $<1.045$ & $>12.736$ \\
Expectativa de vida (años) & 61 & 79 \\
Mortalidad infantil (muertes/1.000 nacidos vivos) & 56 & 5 \\
Prevalencia de desnutrición (\%) & 21,8 & 1,4 \\
Mortalidad materna (muertes/100.000 nacidos vivos) & 450 & 17 \\
Incidencia de malaria (casos en un año/100.000 individuos) & 11.165 & 31 \\
\hline Prevalencia de tuberculosis (casos/100.000 individuos) & 352 & 265 \\
\hline Prevalencia de VIH (casos/100.000 individuos) & 1.428 & 0 \\
\hline
\end{tabular}

PBR: Países de Bajos Recursos; PAR: Países de Altos Recursos: PIB: Producto Interno Bruto, USD: Dólares Estadounidenses; VIH: Virus de Inmunodeficiencia Humana.

lucrados con los propios beneficiados, buscando el desarrollo de capacidades en el capital humano local ${ }^{4}$. Por último, los OM, se definen como "un grupo de individuos que viajan a un país extranjero, con el fin de entregar servicios médicos, educación o implementos a la gente de aquel país" ". Hoy en día, existen aproximadamente 4.278 ONGs relacionadas con el área de salud y se estima que 249.000 individuos trabajan en estos proyectos alrededor del mundo ${ }^{6}$.

Dado el desarrollo económico, social y académico logrado en nuestro país, hemos sido parte de un verdadero cambio de paradigma en cuanto a la CI. En los últimos 20 años, nos hemos transformado de ser un país receptor a ser país exportador de CI. Así lo demuestran estadísticas nacionales de la Agencia de Cooperación Chilena para el Desarrollo (AGCID Chile) $)^{3}$, y la generación de ONGs ligadas a la cooperación, como América Solidaria ${ }^{7}$ o África Dream ${ }^{8}$, entre otras.

Debido a la relevancia del tema, el aumento de literatura científica, y el desarrollo de instancias de colaboración desde nuestro país ${ }^{5,7,8}$, nos planteamos como objetivo realizar una revisión narrativa sobre la CI. Inicialmente describiremos la $\mathrm{AH}$ y CD, para luego enfocarnos en los OM. Los clasificaremos según sus características, evaluaremos el impacto de las intervenciones, los riesgos y controversias asociados a ellas y posibles proyecciones futuras, basados en la mejor evidencia disponible y nuestra propia experiencia.

\section{Caracterización de la CI}

Los desastres naturales o episodios de violencia armada que afectan a una región producen una situación especial, en que aumentan las necesidades de atención médica de la población y, muchas veces, el sistema de salud se encuentra mermado (daños en la infraestructura o disminución de personal calificado). Así se produce un desbalance entre la demanda y la oferta de atención sanitaria, ya sea por un deterioro incidental del funcionamiento del sistema de salud, por un aumento transitorio de las necesidades de atención sanitaria, o por una combinación de ambas situaciones. Los OM de grupos individuales no son capaces de hacer frente a situaciones como estas, las que son abordadas por organizaciones más complejas, como cuerpos sanitarios militares, instituciones como el Comité Internacional de la Cruz Roja (CICR) u ONGs como Médicos Sin Fronteras $(\mathrm{MSF})^{5}$. Estas instituciones son capaces de gestionar proyectos de $\mathrm{AH}$ con la magnitud necesaria, proporcionando la logística necesaria para construir hospitales de campaña u optimizar el trabajo en hospitales locales, y aportando profesionales e insumos. Si bien el personal sanitario extranjero permanece por un período de tiempo acotado, pueden percibir remuneraciones y rota por un tiempo variable; en ocasiones el proyecto incluye a personal sanitario local estable y tiene la duración suficiente para permitir al sistema 
de salud local recuperarse y volver a satisfacer la demanda sanitaria de manera independiente. A modo de ejemplo, durante el año 2015, MSF atendió a 594.900 pacientes hospitalizados, entregó el tratamiento a 230.400 pacientes portadores de VIH, asistió 219.300 partos o cesáreas, vacunó a 1.537.400 personas contra sarampión, entre otros 9 .

Sin embargo, no son extraños los casos en que el sistema local no es capaz de independizarse, porque, además del daño causado por el desastre natural o la violencia armada, se encuentran sufriendo de una carencia crónica de recursos, personal e insumos. Es por esto que se ha establecido un modelo distinto a la AH, denominado CD. En este, se busca alcanzar el destete progresivo del apoyo internacional, logrando el establecimiento de estructuras e instituciones eficientes y autosustentables dentro del país. Diversas experiencias han demostrado su utilidad , $^{4,10,11,12}$.

Por otra parte, los OM se originan por la constatación de una población con alguna necesidad sanitaria insatisfecha ${ }^{13}$. Para subsanar este problema se organizan equipos de personal de salud para resolver casos seleccionados, por lo general patologías quirúrgicas, que no pueden ser resueltos por el sistema local ${ }^{5,13,14}$. Este tipo de misiones tienen las ventajas de la optimización del trabajo, porque se trata de equipos compenetrados (aunque muchos pueden no conocerse con anticipación), la posibilidad de resolver patologías complejas e intentar asegurar un nivel de atención estándar a los pacientes.

Cabe destacar que un número significativo de estos OM son organizados por instituciones académicas de PAR asociadas a instituciones educacionales locales de PBR. De esta forma, se promueve el intercambio bilateral entre estudiantes y docentes, reportando beneficios para ambos grupos. Por un lado, las instituciones locales reciben apoyo económico, académico y técnico, y algunos de sus alumnos pueden especializarse o perfeccionar sus conocimientos en el extranjero, para luego importarlos a su país de origen; por otra parte, los estudiantes de PAR pueden conocer nuevas realidades, aprender soluciones creativas para problemas cotidianos, y tomar conciencia de los problemas de la salud global.

Una de las principales interrogantes de estos $\mathrm{OM}$ es el real impacto que tienen $\mathrm{y}$, debido a la gran cantidad de grupos y a la enorme heteroge- neidad en el trabajo que estos desarrollan, es difícil hacer una evaluación sistematizada. Martiniuk et $\mathrm{al}^{14}$ realizaron una revisión sistemática de la literatura evaluando los OM. En esta refieren que los países que más OM envían son Estados Unidos de Norteamérica, Canadá, Reino Unido y Australia, siendo las regiones beneficiadas de este apoyo, principalmente, África, América Central y Asia. Sin embargo, la estructura, calidad y objetivos de cada OM son distintos, y existen muchas áreas que merecen una revisión más profunda.

\section{Riesgos y controversias de los OM}

El aumento progresivo en el desarrollo de distintos tipos de CI ha generado debate sobre el real impacto y riesgos que esconden los distintos tipos de proyectos en la actualidad.

A pesar del carácter solidario imperante en este tipo de actividades, existen aspectos negativos descritos en la literatura, asociados principalmente a la implementación, contextualización e impacto. Maki et al ${ }^{15}$ resumen los problemas de algunos $\mathrm{OM}$ en la siguiente frase: "la falta de seguimiento, la pobre relación con el sistema de salud local y la escasa sustentabilidad pueden desafiar las buenas intenciones de las misiones". Martiniuk et al describen las principales críticas encontradas en la literatura, luego de una revisión sistemática, destacando la sustentabilidad, costo-efectividad, seguridad y consideraciones éticas relacionadas con la praxis ${ }^{14}$. Caldron et al incorporan también la profesionalización como elemento a considerar ${ }^{5}$. Intentando simplificar las principales controversias y riesgos implicados en los OM, las analizaremos en los siguientes subgrupos.

\section{Sustentabilidad/Costo efectividad}

Una de las principales críticas a los OM de corta duración (duración menor de 8 semanas) es el bajo impacto y ausencia de mantención del efecto a largo plazo. Múltiples factores inciden en estos fenómenos, como la falta de comunicación con los agentes locales, poca comprensión del medio y sus necesidades, falta de integración local y transferencia de conocimientos. Asimismo, la escasez de métodos de evaluación comprehensivos y programas de mejoramiento continuo de los OM hacen de la sustentabilidad un foco de controversia ${ }^{13}$. 
Muy ligado a la sustentabilidad, aparece el concepto de costo-efectividad. Actualmente no se tiene registro público sobre el gasto promedio de los distintos OM. Abdullah ${ }^{16}$ cuestiona el impacto de su OM en Ghana, al comparar la inversión total de 30.000 USD (seguros, alojamientos, vacunas, pasajes y alimentación para 10 voluntarios), cuando la construcción de un hospital en ese país, con 30 camas, tiene un costo aproximado de 60.000 USD.

En 1993, el Banco Mundial estableció un monto de 150 dólares norteamericanos por cada año de vida ajustado por discapacidad (DALY) como variable para evaluar costo-efectividad en proyectos de $\mathrm{CI}^{17}$. Asimismo, dado el gran número de ONGs con objetivos similares ${ }^{18}$ y la escasa coordinación que puede existir entre ellas, en ocasiones los esfuerzos se pueden duplicar, anularse o pueden utilizarse los recursos disponibles de manera subóptima. Sykes ${ }^{13}$ identificó sólo 3 estudios donde se registró la evaluación en términos de costo efectividad, y en uno de ellos casi se triplicó la cifra establecida por el Banco Mundial.

Sin embargo, esta crítica puede ser más aplicable a OM llevados a cabo por voluntarios individuales y organizaciones pequeñas que al trabajo de grandes ONGs en proyectos de $\mathrm{AH}$ y $\mathrm{CD}$, las que habitualmente deben presentar balances financieros y reportes de actividades a sus fuentes de financiamiento. Los proyectos de CD tienen como objetivo central que las instituciones locales dejen de depender de la ayuda internacional, por lo que las consideraciones de sustentabilidad debiesen estar bien enfrentadas por el proyecto mismo. Si bien el criterio monetario no debe ser el único utilizado durante la evaluación de los OM, la correcta evaluación sobre costo-efectividad en este tipo de proyectos aún está pendiente.

\section{Profesionalización/Seguridad}

Otro aspecto de gran preocupación es la calidad y efectividad de la actividad del equipo médico. Existen dudas sobre la preparación de los equipos de PAR para desenvolverse en un terreno desconocido y completamente distinto al escenario de formación o de práctica habitual. Esto podría generar un aumento no evaluado de complicaciones médicas no previstas que, junto a la posible falta de comprensión del medio y sus recursos, o la sensación de abandono luego de fin del OM, podría generar un aumento de la des- confianza por parte de la población local hacia los $\mathrm{OM}^{18}$. Además, la falta de preparación previa, integración con el equipo local y desarrollo de un plan estratégico puede llevar a generar proyectos mal desarrollados y ejecutados inadecuadamente ${ }^{18}$.

Por otra parte, factores como los déficits de leyes o formalidades de regulación harían de estas instancias un buen escenario para que profesionales menos preparados en sus países de origen, interesados en mejorar sus habilidades técnicas, o motivados por el "turismo médico", participen de este tipo de actividades ${ }^{14}$.

La profesionalización es un concepto en desarrollo en el ámbito de la CI, y donde las grandes ONGs han desarrollado programas de mejoramiento continuo. Es necesario optimizar las distintas vías para regularizar, estandarizar y asegurar la calidad de atención en los países receptores de este tipo de actividades. Para lograrlo, se debe potenciar el desarrollo de guías clínicas, programa de preparación para los voluntarios y regulaciones al accionar médico en este tipo de actividades ${ }^{18}$.

También es necesario considerar los riesgos físicos y mentales a los que se expone el voluntario. La correcta implementación de programas de vacunación y quimioprofilaxis son necesarios para disminuir al mínimo los riesgos sanitarios, como la exposición a enfermedades infecto-contagiosas y accidentes corto-punzantes. A su vez, los conflictos bélicos o accidentes por falta de normativas de seguridad corresponden a riesgos inherentes de situaciones políticas inestables o precarias. Por último, existe el riesgo que las experiencias vividas generen síntomas psiquiátricos, como trastornos del ánimo y estrés postraumático. Este riesgo debe ser conocido y acogido por el equipo, asegurando acompañamiento y tratamiento profesional en caso necesario, tanto en terreno como una vez concluido los OM.

\section{Consideraciones éticas}

Tradicionalmente, se asume que los $\mathrm{OM}$, la $\mathrm{AH}$ y la CD se caracterizan por su esencia solidaria y que se rigen por valores éticos incuestionables. Asimismo, se asume que el actuar médico no se condiciona según las circunstancias ni el lugar, primando el respeto y bienestar de los pacientes. Sin embargo, el contexto, los recursos, el lenguaje y las barreras culturales relacionadas con la ayuda 
humanitaria, convierten a estas premisas en un verdadero desafío.

En situaciones de CI, donde la población objetivo es inherentemente vulnerable, se corre el riesgo de explotación a distintos niveles, no siempre consciente. Desde la falta de comprensión del lenguaje para explicitar beneficios y riesgos de tratamientos, hasta la vulneración del derecho de autonomía, por ejemplo, al no considerar, muchas veces, el proceso de consentimiento informado como parte del accionar médico en una cultura distinta, hacen de los OM objetivo de reflexión desde la mirada ética.

Por otro lado, en relación a la seguridad y bienestar de la población objetivo, variables como el gran número de pacientes, el tiempo de duración limitado de la mayoría de los OM y, en gran parte de los casos, la escasez de recursos técnicos y humanos, ponen en jaque algunas consideraciones éticas asociadas a la buena praxis profesional. Stone et $\mathrm{al}^{19}$ describen cómo el volumen de pacientes podría afectar a la transparencia y equidad percibida por la comunidad local, al tomar decisiones basadas en la categorización, sin criterios establecidos en conjunto. Hacen también mención a la valoración ética del seguimiento y manejo de las complicaciones asociadas a la atención de salud como un mínimo a conseguir. Se cuestiona que estándares de atención que no son válidos en PAR sean practicados, $y$ hasta promovidos, durante un OM. Bonner et $\mathrm{al}^{20}$, en relación a un estudio cualitativo realizado en Honduras sobre ética y OM de corta duración, plantean el conflicto de proveer intervenciones por debajo de los estándares aceptables de cuidado, contrastándolo con la decisión de no realizar ninguna acción. Todo esto, en parte, ejemplifica la falta de sustento del "minimalismo ético"21 atribuido a este tipo de actividades, reflejando la necesidad de hacer conscientes las múltiples dimensiones involucradas.

Paralelamente, junto con el reconocimiento de estas falencias, se abre la puerta para discutir qué rol les compete a los distintos OM, cuando muchas veces, son testigos únicos de situaciones extremas, tanto desde el punto de vista humano como profesional. Por ejemplo, ¿qué sucede en aquellos casos donde se es testigo de procedimientos complejos, éticamente discutibles (la mutilación genital femenina, entre otros)? ¿Qué ocurre cuando se es testigo de violaciones a los derechos humanos por parte de un régimen político o por el mismo personal de salud local? Se hacen perentorios la reflexión y los cuestionamientos sobre los límites éticos y responsabilidades involucradas.

Todos estos problemas han motivado que ONGs y agencias de gran envergadura enfrenten el problema por medio del desarrollo de iniciativas como el "Proyecto Esfera", con el fin de mejorar la calidad de la asistencia humanitaria y promover el actuar responsable de las ONGs hacia los beneficiados y benefactores ${ }^{22}$, especialmente para la $\mathrm{AH}$. Sin embargo, las instituciones que hacen $\mathrm{OM}$ no siempre conocen este marco de referencia ético.

\section{Proyecciones futuras}

En el escenario actual, y evaluando las proyecciones en un futuro próximo, es importante reflexionar sobre cómo y hacia dónde nos estamos dirigiendo.

Primeramente, es necesario reflexionar sobre los objetivos implicados en los distintos OM y la forma de evaluar continuamente los procesos involucrados. Para las actividades que involucren un trabajo a largo plazo, se debiese priorizar actividades que permitan y faciliten el empoderamiento local, con construcción de objetivos en conjunto, para evitar, en su fin último, la dependencia a la presencia externa ${ }^{18}$. Es de responsabilidad de cada OM, AH y CD analizar y criticar su impacto en la población objetivo, estableciendo, a priori, diferentes mecanismos de evaluación para esto.

Por otra parte, la actual escasez de registros sobre los distintos OM y sus implicancias abre un espacio para generar nuevas instancias de discusión y desarrollo, lo cual debe ser plasmado en publicaciones, cursos, conferencias y congresos que permitan el crecimiento de esta área ${ }^{5,23}$.

Paralelamente, creemos necesaria la revisión de los procesos de postulación y acompañamiento de voluntarios. Los participantes cobran un papel extremadamente relevante en la correcta implementación de los objetivos propuestos por cada proyecto de OM, AH y CD. Se hace importante generar procesos de postulación transparentes y acordes a las necesidades de cada proyecto, sumado a espacios de reflexión, acompañamiento y preparación, previo y posterior a la experiencia en terreno. De esta forma, disminuir el impacto inicial e intentar asegurar la mejor comprensión del medio local. 
Por último, en relación a los riesgos e implicancias médico-legales, se debe abrir el debate sobre regulaciones internacionales que aseguren estándares mínimos para la praxis profesional. Además, generar conciencia sobre la obligación que compete al voluntario, de conocer el funcionamiento del sistema local y sus leyes ${ }^{19,23,24}$.

\section{Conclusión}

Los $\mathrm{OM}, \mathrm{AH}$ y $\mathrm{CD}$ corresponden a un fenómeno en exponencial crecimiento, tanto en los PAR como en nuestro país. Este crecimiento, sin embargo, debe ir acoplado a una profesionalización y un desarrollo formal de la disciplina. Proyectos bajo el paradigma de la cooperación para el desarrollo apuntan a enriquecer a la cultura local y la transferencia de conocimientos y herramientas a los propios actores, para que, en un futuro próximo, este tipo de actividades no sean necesarias y estos actores sean capaces de resolver sus propios desafíos sanitarios.

\section{Referencias}

1. International Health Conference. Constitution of the World Health Organization 1946. Bull World Health Organ 2002; 80 (12): 983-4.

2. Organización Mundial de la Salud. Estadísticas Mundiales de Salud 2014. Disponible en internet, http://www. who.int/gho/publications/world_health_statistics/2014/ es/ (Acceso el 19 de septiembre de 2016).

3. Agencia Chilena para la Cooperación y Desarrollo. Quienes Somos. Disponible en internet, http://www. agci.cl/index.php/acerca-de-agci/quienes-somos (Acceso el 19 de septiembre de 2016).

4. Lipnick M, Mijumbi C, Dubowitz G, Kaggwa S, Goetz L, Mabweijano, et al. Surgery and anesthesia capacity-building in resource-poor settings: description of an ongoing academic partnership in Uganda. World J Surg 2013; 37 (3): 488-97.

5. Caldron PH, Impens A, Pavlova M, Groot W. A systematic review of social, economic and diplomatic aspects of short-term medical missions. BMC Health Services Research 2015; 15: 380.

6. Harvey P, Stoddard A, Harmer A, Taylor G. State of the humanitarian system report. ALNAP. 2015, Disponible en Internet, www.alnap.org (Acceso el 19 de septiembre de 2016).

7. Fundación América Solidaria. Nuestra Historia. Dis- ponible en internet, http://www.americasolidaria.org/ conocenos/nuestra-historia/ (Acceso el 19 de septiembre de 2016).

8. Fundación Africa Dream. Quienes Somos. Disponible en internet, http://africadream.cl/wp12/index.php/ que-hacemos/ (Acceso el 19 de septiembre de 2016).

9. Médicos Sin Fronteras. MSF International Activity Report 2015. Disponible en internet, http://www.msf.org/ sites/msf.org/files/international_activity_report_2015_ en_2nd_ed_0.pdf (Acceso el 2 de junio de 2017).

10. Qureshi JS, Samuel J, Lee C, Caims B, Shores C, Charles A. Surgery and global public health: the UNC-Malawi surgical initiative as a model for sustainable collaboration. World J Surg 2011; 35: 17-21.

11. Ozgediz D, Wang J, Jayaraman S, Ayzengart A, Jamshidi R, Lipnick M, et al. Surgical training and global health: initial results of a 5 year partnership with a surgical training program in a low-income country. Arch Surg 2008; 143: 860-5.

12. Riviello R, Ozgediz D, Hsia RY Azzie G, Newton M, Tarpley J. Role of collaborative academic partnerships in surgical training, education, and provision. World J Surg 2010; 34: 459-65.

13. Sykes KJ. Short-Term Medical Service Trips: A Systematic Review of the Evidence. Am J Public Health 2014; 104 (7): e38-e48.

14. Martiniuk ALC, Manouchehrian M, Negin JAZwi AB. Brain Gains: a literature review of medical missions to low and middle-income countries. BMC Health Services Research 2012; 12: 134.

15. Maki J, Qualls M, White B, Kleefield S, Crone R. Health impact assessment and short-term medical missions: a methods study to evaluate quality of care. BMC Health Serv Res 2008; 8: 121.

16. Abdullah F. Perspective of West Africa: why bother to "mission"? Arch Surg 2008; 143 (8): 728-9.

17. Moon W, Perry H, Baek RM. Is international volunteer surgery for cleft lip and cleft palate a cost-effective and justifiable intervention? A case study from East Asia. World J Surg 2012; 36 (12): 2819-30.

18. Fisher Q, Fisher G. The Case for Collaboration Among Humanitarian Surgical Programs in Low Resource Countries. Anesth Analg 2014; 118 (2): 448-53.

19. Stone GS, Olson KR. The Ethics of Medical Volunteerism. Med Clin North Am 2016; 100 (2): 237-46.

20. Bonner J, Hopper J, Gore Martin L, Caldwell P. Pilot Surveys of Ethics and Short-Term Medical Mission Work: University of Wyoming Agua Salada Clinic, Honduras. Univers J Public Health 2013; 1 (3): 86-96.

21. Wall LL, Arrowsmith SD, Lassey AT, Danso K. Humanitarian ventures or 'fistula tourism?': the ethical perils 
of pelvic surgery in the developing world. Int Urogynecol J Pelvic Floor Dysfunct 2006; 17 (6): 559-62.

22. Proyecto Esfera. Acerca de Esfera. Disponible en internet, http://www.sphereproject.org/sphere/es/ (Acceso el 19 de septiembre de 2016).

23. Withers M, Press D, Wipfli H, McCool J, Chan CC,
Jimba $\mathrm{M}$, et al. Training the next generation of global health experts: experiences and recommendations from Pacific Rim universities. Global Health 2016; 23; 12 (1): 34.

24. Casey KM. The Global Impact of Surgical Volunteerism. Surg Clin North Am 2007; 87 (4): 949-60. 